\title{
NUMERICAL INVESTIGATION OF THE LEAN EFFECTS IN CENTRIFUGAL COMPRESSORS
}

\author{
F. Merli* - P. Gaetani \\ Dipartimento di Energia, Politecnico di Milano, Via Lambruschini, 4, 20156 Milano - Italy \\ filippo.merli@mail.polimi.it; paolo.gaetani@polimi.it \\ www.lfm.polimi.it \\ * currently at Institute for Thermal Turbomachinery and Machine Dynamics, \\ Graz University of Technology - Austria
}

\begin{abstract}
The continuous need of performance improvements in the centrifugal compressor technology for industrial and aeronautical applications, as well as the modern CAD techniques allow and ask for new impeller designs.

In this paper a numerical analysis of opposite lean angles on the rotor blades is carried out. These methodologies are widely applied to axial turbomachines, but few papers in the open literature can be found on centrifugal compressors.

Blade lean effects on the rotor outlet flow and the vaneless diffuser performances are discussed. Some of the blades have been tested for 3 tip clearances and 3 flow rates to get a deeper understanding of the involved phenomena.

Results show a slight dependence of the rotor efficiency on rotor blade lean; notwithstanding, the local flow field at the rotor outlet presents different patterns that affect the flow evolution in the vaneless diffuser and its performances.
\end{abstract}

\section{KEYWORDS}

\section{CENTRIFUGAL COMPRESSOR, LEAN, TIP CLEARANCE, CFD}

\begin{tabular}{llll} 
NOMENCLATURE & \multicolumn{2}{l}{} \\
$\mathrm{b}$ & blade height & $\sigma$ & \multicolumn{1}{l}{ lean angle } \\
$\mathrm{C}_{\mathrm{p}}$ & pressure recovery coefficient & $\omega$ & rotational speed \\
$\mathrm{D}, \mathrm{R}$ & diameter, radius & \multicolumn{2}{l}{ Subscripts: } \\
$\dot{\mathrm{m}}$ & mass flow rate & 1 & impeller inlet \\
$\mathrm{P}$ & pressure & 2 & impeller outlet \\
$\mathrm{PS}, \mathrm{SS}$ & pressure side / suction side & 3 & diffuser outlet \\
$\mathrm{T}$ & temperature & $\mathrm{IN}, \mathrm{OUT}$ & domain inlet / outlet \\
$\mathrm{V}, \mathrm{W}$ & absolute /relative velocity & $\mathrm{LE}, \mathrm{TE}$ & leading / trailing edge \\
$\alpha, \beta$ & absolute / relative flow angle & $\mathrm{SW}$ & streamwise \\
$\delta$ & tip clearance & $\mathrm{T}$ & total
\end{tabular}

\section{INTRODUCTION}

Centrifugal compressors are commonly used in many applications, such as the Oil\&Gas industry, the automotive sector and some aero-engines, heating \& cooling technologies, as described in Japikse (1996) and Cumpsty (1989).

The main reasons for radial design success are the reduced dimensions compared to the axial machines, the intrinsic higher reliability, its fairly large operational range at fixed driver speed which can be widened by applying adjustable IGVs and a generally high efficiency. In particular the efficiency is nowadays of paramount importance in the context of environmental friendly industrial processes and operational costs reduction. 
Within this frame, designers have a number of options to boost the efficiency, like blade shape optimisation, blade-casing clearance sizing, inlet/outlet area ratio choice, diffuser design. Modern $\mathrm{CAD}$ tools and manufacturing technologies also allow for complex turbomachinery geometries, supporting the performance enhancement.

These aspects can be directly investigated by means of experiments and CFD, once its model is validated against accurate experimental results. Many experimental works have been proposed in the open literature in these last decades: studies on the impeller flow, since the pioneer work of Krain (1981), evidenced the 3D character of the Jet\&Wake feature (among others: Ziegler et al. 2003a, b; Schleer et al., 2004; Toni et al., 2010; Gaetani et al., 2011 and 2012). In particular, the dissipative region - connected to the action of the secondary flows, whose magnitude and extension depends on the operational point and on the geometry - accumulates on the tip/suction side corner of the impeller channel at the outlet section. Such flows may have a severe impact on the diffuser performances and are expected to be modulated by the impeller blade lean. CFD was also intensively applied to study possible optimal configurations in terms of geometrical and operational parameters.

In this paper the focus is on the blade shape, specifically on the blade lean angle and on the casing-blade tip clearance, which have been numerically analysed in an unshrouded impeller for different flow rates values. To gain reliable results, the CFD model has been previously validated against the experimental data reported in Gaetani et al. (2011) for the design condition and (2012) for the off-design. The reference rotor is representative of industrial compressors and, accordingly, results are expected to be of general interest.

The goal is to improve the understanding of the flow features affected by the lean angle and its interaction with the tip clearance. This work considers primarily the concepts about lean widely discussed for axial turbomachines (see for example Denton et al., 1999; D’Ippolito et al., 2011; Rosic et al., 2012) and less frequently for radial machines (Van den Braembussche, 2006; He and Zheng, 2016). As for the tip clearance, among the works found in literature Baines and Whitfield (1990), Brasz (1998), Baskharone (2006), Hong and Abhari (2012) are brought to the readers' attention. To take into account for this parameter in the 1D design process, the Daily\&Nece correlation is often applied.

First the rotor flow field is analysed for the reference flow rate; then the impact of the rotor outlet on the vaneless diffuser and its control, operated by leaning the blade, is discussed. Finally, results at different flow rates and tip clearance are also presented and commented.

\section{BLADE MODELLING}

The reference blade, as well as the blade channel shape, were taken from an existing compressor in use at LFM. The main characteristics of the geometry adopted for numerical simulations (impeller + vaneless diffuser) are listed in Tab. 1. As shown by the geometric angle $\beta_{g}$ meridional evolution (Fig. 1), the blade CAD model is twisted in the leading region, while in the last $5 \%$ of its meridional extent $\beta_{g}=-24.9^{\circ}=$ const along the span.

\begin{tabular}{l|c}
\hline \multicolumn{1}{c|}{ IMPELLER } & VANELESS DIFFUSER \\
\hline$N$. Blades $=16$ & $b_{\text {diff }}=17.9 \mathrm{~mm}$ \\
$b_{2}=17.4 \mathrm{~mm}$ & $D_{3}=640 \mathrm{~mm}$ \\
$\delta=0.5 \mathrm{~mm}$ & \\
$D_{1, t i p}=262 \mathrm{~mm}$ & \\
$D_{2}=400 \mathrm{~mm}$ & \\
\hline
\end{tabular}

Tab. 1: Main geometrical characteristics

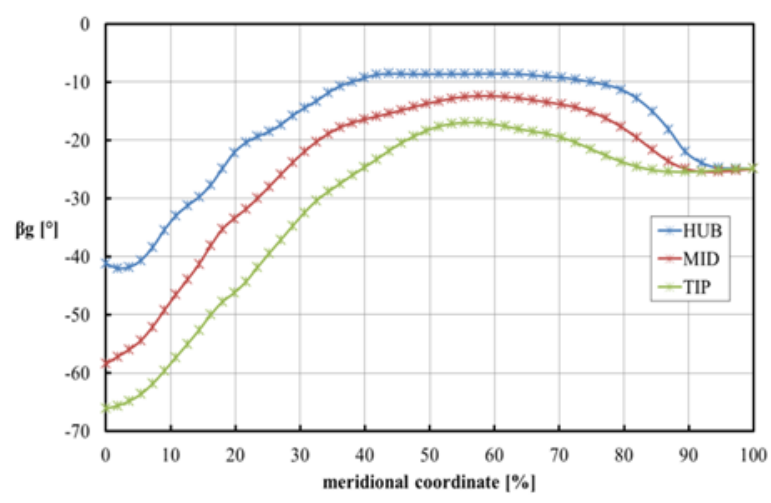

Fig. 1: Blade angle distribution along the meridional coordinate 


\section{Geometry}

The lean angle $\sigma$ is defined as the angle between the normal to the hub wall and the stacking line of the 2D blade sections, with $\sigma>$ 0 meaning that the pressure side generates an acute angle with the blade-to-blade surfaces. The leaned contour was created by shifting tangentially the tip section of the original blade, keeping the same thickness and geometric angle meridional evolution. Adapting the relations proposed by Howard and Ashrafizaadeh (1994):

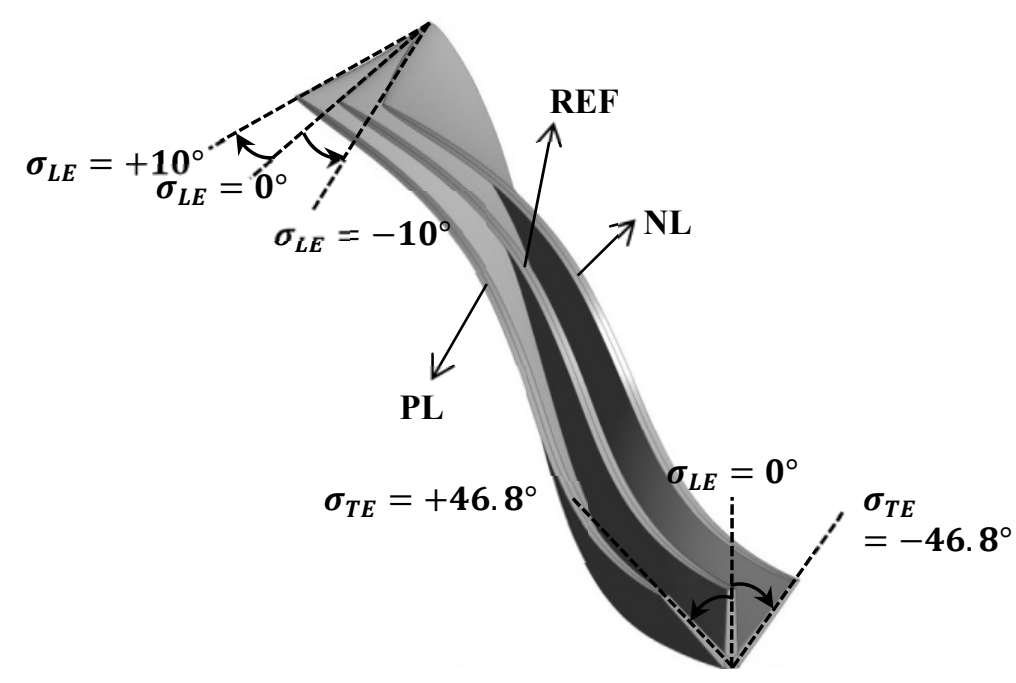

Fig. 2: Reference and leaned blades

$$
\sigma_{L E}=\frac{r_{1, t i p} \sin (\Delta \theta)}{\sqrt{r_{1, t i p}^{2}+r_{1, h u b}^{2}-2 r_{1, t i p} r_{1, h u b} \cos (\Delta \theta)}} \quad \text { and } \sigma_{T E}=\frac{2 r_{2} \sin \left(\frac{\Delta \theta}{2}\right)}{b_{2, r e f}}
$$

a constant tangential displacement $\Delta \theta$ of the blade tip was set in order to generate two leaned geometries, respectively with $\sigma_{L E}=10^{\circ}$ and $\sigma_{L E}=-10^{\circ}$, corresponding to $\sigma_{T E}=46.8^{\circ}$ and $\sigma_{T E}=-46.8^{\circ}$, while the reference blade has $\sigma_{L E}=$ $\sigma_{T E}=0^{\circ}$ (Fig. 2). As the blade points tangential position $\theta$ along the meridional coordinate $m$ and the geometric angle are related by (Van den Braembussche, 2006):

$$
\tan \beta_{g}=r \frac{d \theta}{d m}
$$

\begin{tabular}{cccc}
\hline Name & $\sigma_{L E}\left[{ }^{\circ}\right]$ & $\sigma_{T E}\left[^{\circ}\right]$ & $\delta[\mathrm{mm}]$ \\
\hline REF & 0 & 0 & 0.5 \\
\hline PL & +10 & +46.8 & 0.5 \\
NL & -10 & -46.8 & 0.5 \\
\hline CL02 & 0 & 0 & 0.2 \\
CL00 & 0 & 0 & 0 \\
\hline
\end{tabular}

Tab. 2: Simulated profiles recap

a constant $\theta$ variation does not affect the blade angle meridional distribution and allows a fair comparison of the different lean cases, preventing possible incidence or deviation effects caused by the geometry variation.

The tip clearance influence on the flow field was studied by comparing the reference blade (which has a constant gap $\delta=0.5 \mathrm{~mm})$ with a small-clearance profile $(\delta=0.2 \mathrm{~mm}$ ) and an "ideal" no-clearance profile $(\delta=0 \mathrm{~mm})$. Finally, Tab. 2 sums up the analysed configurations and their differences in terms of lean angle and tip clearance.

\section{CFD SETUP}

A steady-state RANS approach was considered and the numerical simulations were performed with the commercial code ANSYS $^{\circledR} \quad$ CFX $^{\circledR}$ 18.2. The steady-state option was chosen as no vaned diffuser was considered in the model and for this no stationary-rotating bladed components interaction took place. In addition, no issues were found in terms of convergence, as it could happen when unstable separation occurs in the flow field. Finally, it allowed to calculate a larger number of cases, needed in the foreseen parametric analysis, due to the reduced computational cost compared to URANS.

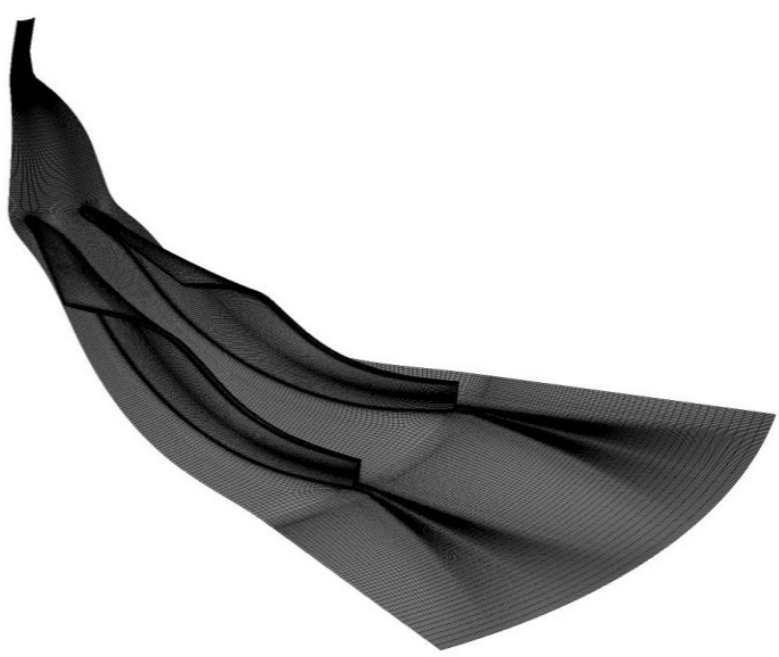

Fig. 3: Reference case computational grid 
The implemented turbulence model was the $k-\omega$ SST (Menter et al., 2003), since it combines the advantages of the Wilcox $k-\omega$ model in terms of near-wall flow (with a better separation prediction) and those of the $k-\varepsilon$ models in freestream turbulence assessment.

\begin{tabular}{cc|cc}
\hline Name & & Case & $\dot{\boldsymbol{m}}_{\text {OUT }}[\mathrm{kg} / \mathbf{s}]$ \\
\hline $\boldsymbol{\omega}[\mathrm{rpm}]$ & 18000 & MIN & 4.35 \\
$\boldsymbol{T}_{\boldsymbol{T}, \boldsymbol{I N}}[\mathrm{K}]$ & 305 & $\mathbf{R E F}$ & 4.93 \\
$\boldsymbol{P}_{\boldsymbol{T}, \boldsymbol{I N}}[\mathrm{bar}]$ & 1.002 & MAX & 5.46 \\
\hline
\end{tabular}

Tab. 3: Boundary conditions

The structured, hexahedral computational grid was created in ANSYS ${ }^{\circledR}$ TurboGrid $^{\mathrm{TM}}$ (Fig. 3) and it was the result of a mesh independence analysis and quality parameters assessment. It has around $1.7 \mathrm{M}$ elm. in the blade passage (about $100 \mathrm{elm}$. spanwise, plus $30 \mathrm{elm}$. in the tip gap) for a

total of $3.8 \mathrm{M}$ elm. in the $0.5 \mathrm{~mm}-0.2 \mathrm{~mm}$ clearance cases and $2.9 \mathrm{M}$ elm. in the no-clearance one. The inflation layer close to solid walls was targeted to have at least 15 cells in the boundary layer and $y_{\text {wall }}^{+} \simeq 1$.

In terms of boundary conditions (Tab. 3), total pressure, total temperature, turbulent intensity and flow direction were imposed at the inlet section, while at the outlet mass flow was assigned. For the reference and leaned cases three mass flow values were considered, corresponding to different operating conditions of the machine: near-surge (MINimum mass flow), near-optimum (REFerence mass flow), near-choke (MAXimum mass flow); on the contrary, reduced clearance cases were studied only in the reference condition. Solid walls were assumed smooth and adiabatic; the interface between the impeller and the diffuser, placed at a radius of $208 \mathrm{~mm}$, is set as frozen rotor.

\section{REFERENCE CASE}

\section{Impeller flow}

To discuss the flow field in the impeller, at first the streamwise relative vorticity plots are presented in Fig. 4, which shows good agreement with the structures described in Van den Braembussche (2006) and found also in He and Zheng (2016). At $6 \%$ of the blade channel length, the counter-clockwise (i.e. negative vorticity) tip clearance vortex (A) starts and interacts with the scraping vortex, the latter arising due to the boundary layer developed on the stationary casing of the open impeller. Negative and positive vorticities are found on suction (SS) and pressure (PS) side
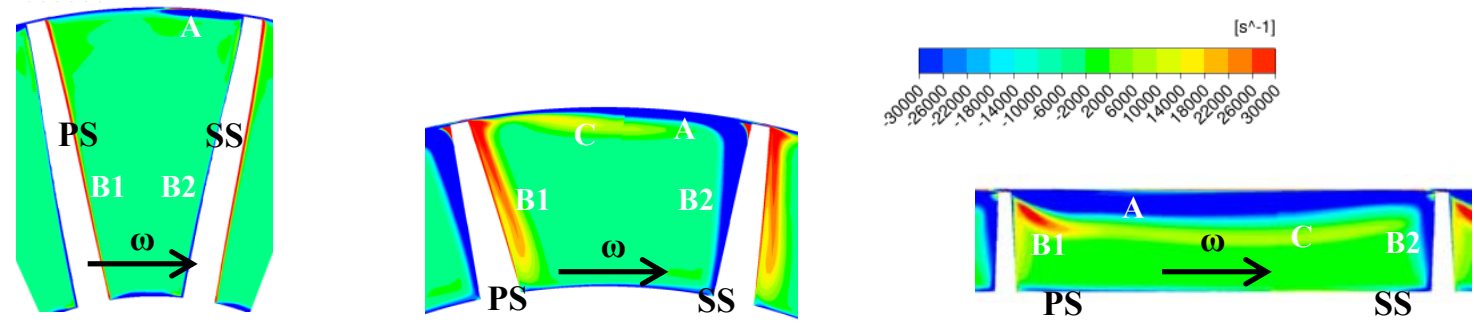

Fig. 4: Streamwise relative vorticity at $6 \%, 50 \%$ of the blade channel length and at $R_{2}$

respectively, as a result of the boundary layer evolution on the blade surfaces (B1, B2).

As the streamwise coordinate proceeds, the blades boundary layer vorticity grows a bit, but the strongest evolution is on the vorticity cores related to the tip clearance and secondary flows evolving in the channel. At the impeller outlet, a wide negative core related to the tip clearance is found close to the casing, contoured by a positive core at $60 \%$ of the blade span, whose positive vorticity is consistent with the passage vortex one (C).

As for losses, the entropy at the impeller outlet (Fig. 5) shows a core in the tip region, consistent with the tip clearance flow and the secondary flows accumulation.

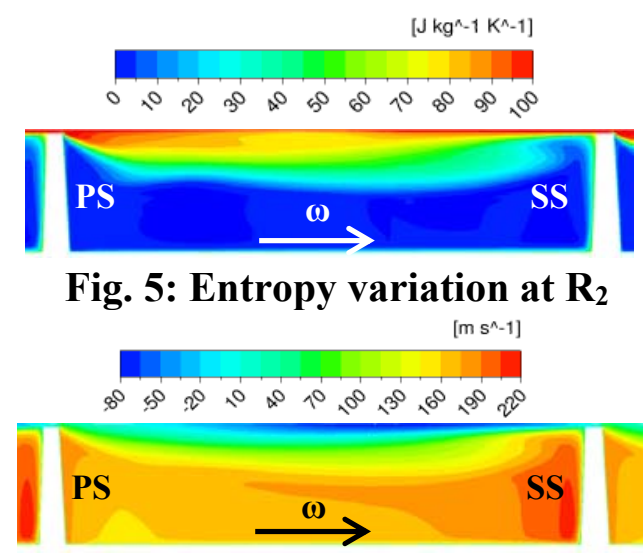

Fig. 6: Radial velocity at $\mathbf{R}_{2}$ 
When the flow rate is of concern, specifically the velocity radial component (Fig. 6), it is of some importance to underline the tip-SS area of reverse flow, that is one of the main causes of a performance decay in the vaneless diffuser. The backflow region is consistent with the high entropy region, thus underlining its cause.
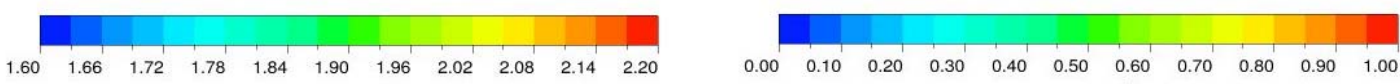

a)

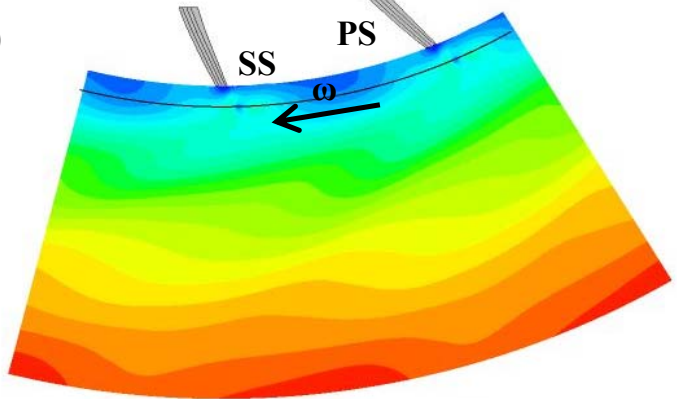

b)

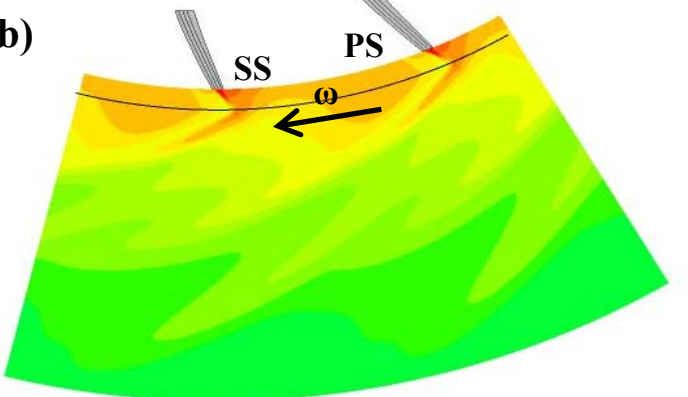

Fig. 7: a) Static pressure and b) Mach number recovery in the vaneless diffuser at midspan

\section{Vaneless diffuser flow}

The vaneless diffuser flow evidences a smooth recovery in the static pressure (Fig. 7a) and reduction in the Mach number (Fig. 7b), both altered by the rotor viscous structures evolution: in fact, on the blade to blade plane at midspan, traces of blade wake and channel secondary flows are still present.

The entropy radial evolution in the diffuser (Fig. 8) shows the smearing of the dissipative cores both in tangential and axial directions, leading to a fairly uniform flow at the diffuser outlet.

In order to track the flow rate evolution in the diffuser and to put in evidence the region of flow reversal, the radial component is plotted in Fig. 9. The tip region is typically affected by reverse flows due to the clearance leak; the hub on the contrary experiences a flow reversal for $\mathrm{R} / \mathrm{R}_{2}>1.5$, when the action of the adverse pressure gradient on a thick boundary layer promotes it.

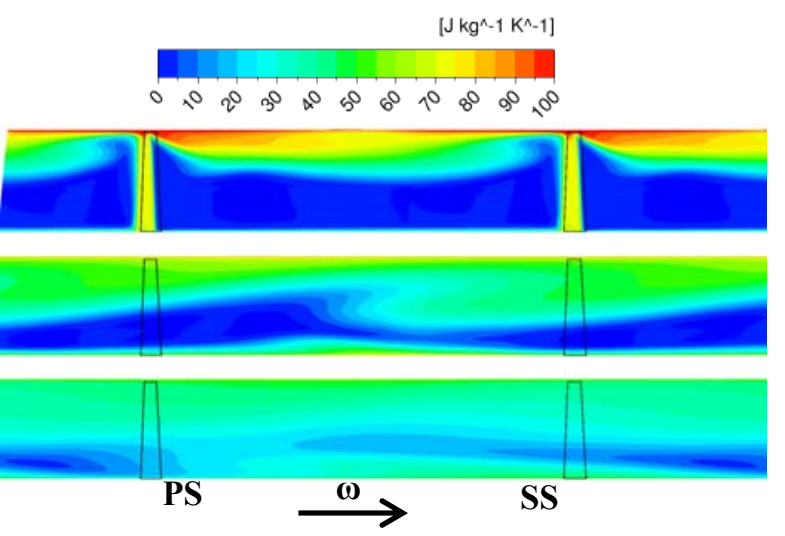

Fig. 8: Entropy evolution

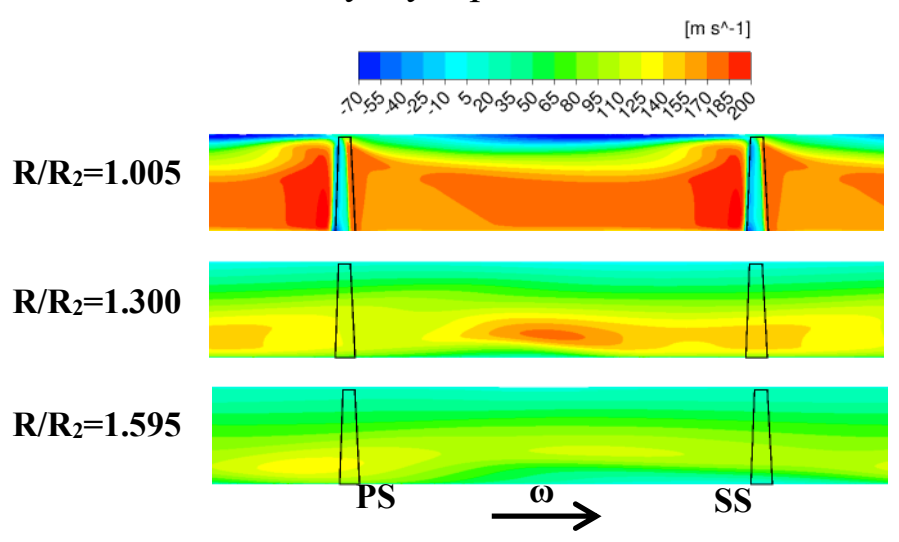

Fig. 9: radial velocity evolution

\section{LEAN EFFECTS AT REFERENCE FLOW RATE}

The positive and negative lean (PL and NL, respectively) influence on the rotor flow field and the impact on the vaneless diffuser downstream are here discussed. In particular, the rotor study has been focused at the discharge section, where $\sigma$ is higher and its action is more evident.

\section{Impeller flow}

According to what found for axial machines by Denton and Xu (1999) or more recently by D'Ippolito et al. (2011), leaned blades affect the pressure distribution in the blade channel, inducing variations in the secondary vortices configuration discussed above. 


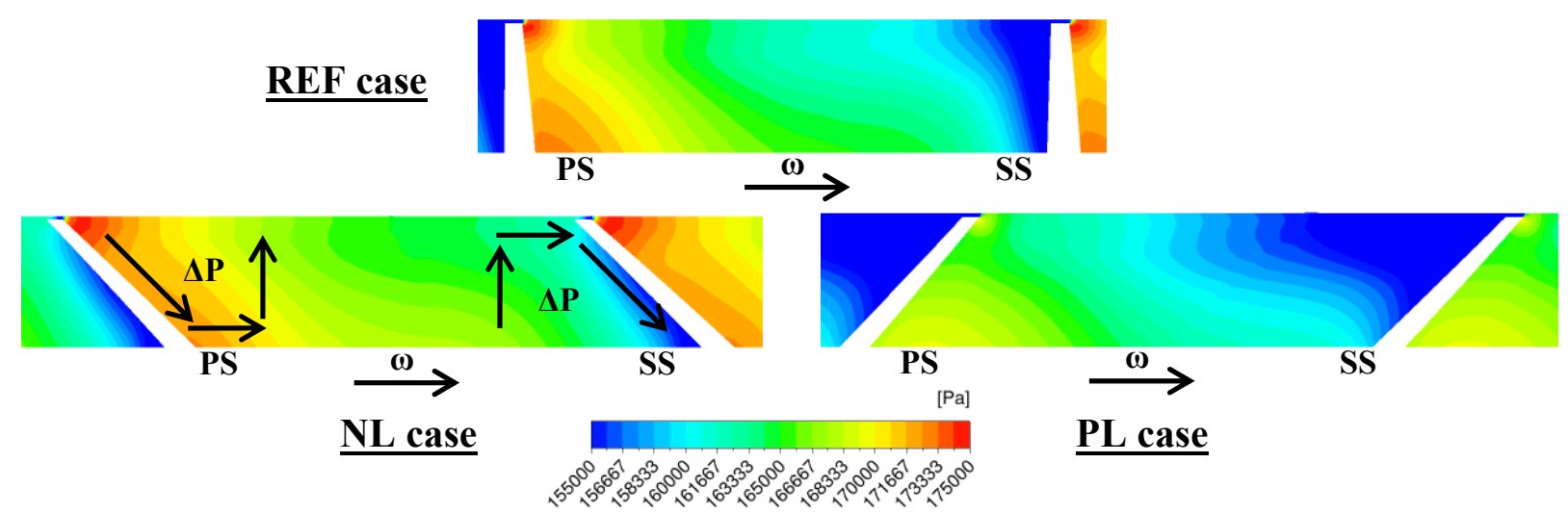

Fig. 10: Pressure distribution at $98 \%$ of the blade channel length for all the cases

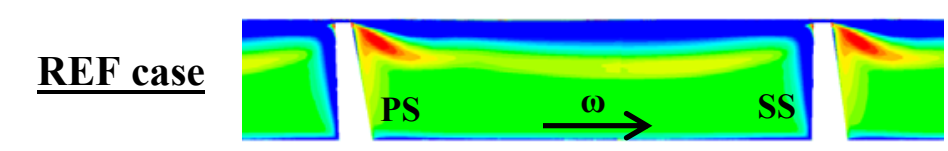

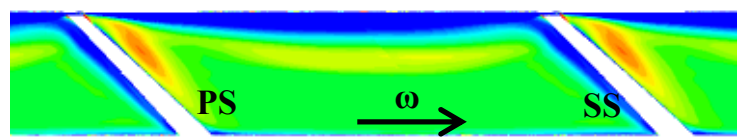

$\underline{\text { NL case }}$

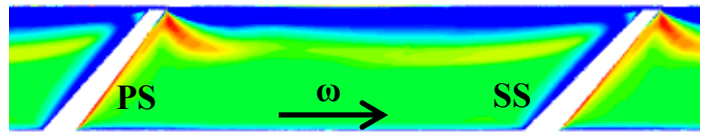

$\underline{\text { PL case }}$

\%일.

Fig. 11: Streamwise relative vorticity distribution at $95 \%$ of the blade channel length for all the cases
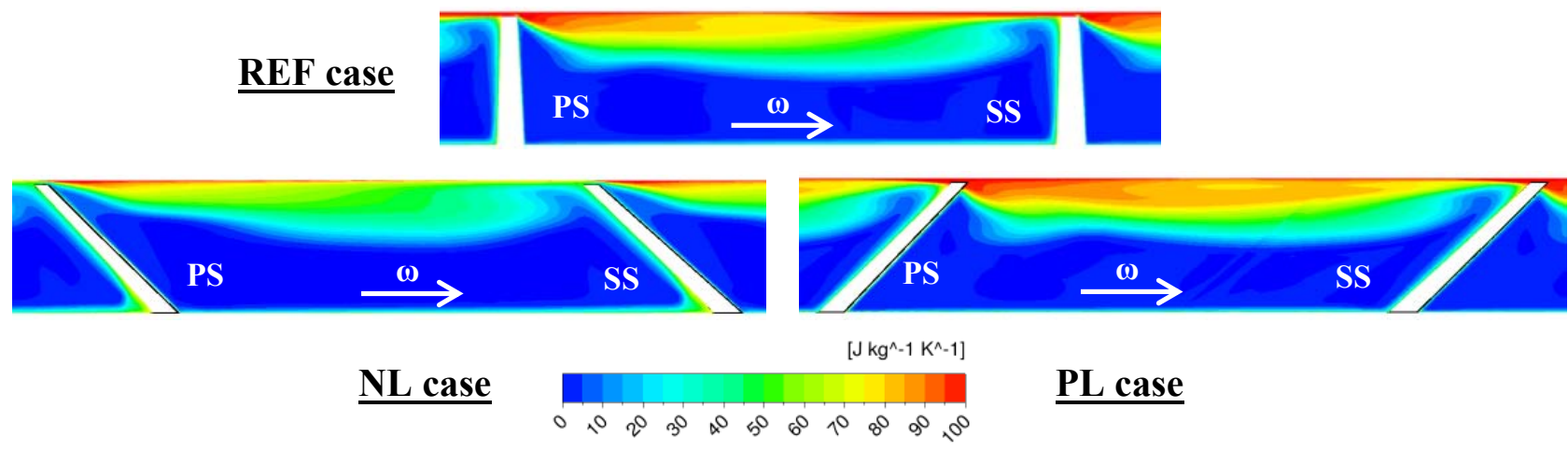

Fig. 12: Entropy distribution at $R_{2}$ for all the cases

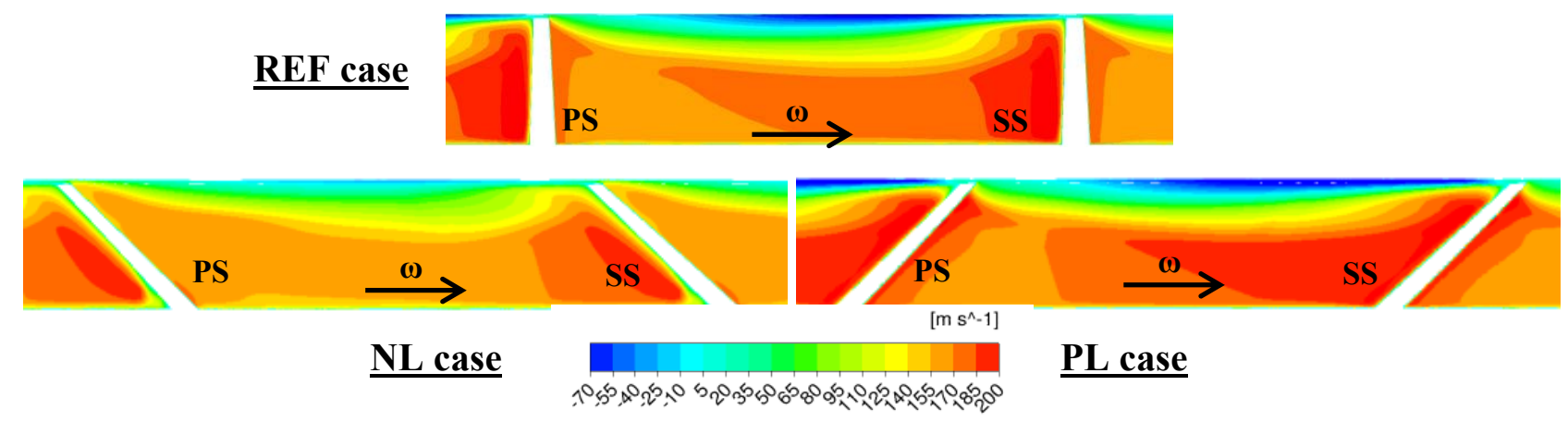

Fig. 13: Radial velocity at $R_{2}$ for all the cases

Close to the blade surface, the isobaric lines in the secondary plane are roughly parallel to the 
blade itself at midspan, while they are getting nearly perpendicular to the end-walls when approaching the tip and hub corners of the channel, due to the isobaric condition in the boundary layer. In the NL case this feature implies a higher pressure at the tip pressure side and suction side corners, as well as a pressure reduction at the hub corners. On the contrary, PL induces higher pressures at the hub corners and lower pressures at the tip ones, if compared to NL and REF cases. Fig. 10 clarifies how a negative lean angle affects the static pressure spanwise distribution at the rotor discharge.

The NL pressure distribution acts against the blade surface vortices, in particular on the PS, leading to a less rotational flow delivered to the diffuser with respect to REF and PL, whose pressure distribution does not show a similar effect (Fig. 10 and Fig. 11).

Another important consequence of the lean angle on the rotor discharge flow regards its influence on the low-momentum fluid close to the upper end-wall, which could explain the reduction of the negative vorticity region size at the casing wall. Due to the inclination of blade, NL tends to push the high-entropy fluid against the casing (especially on the PS, see Fig. 12), while PL pushes it towards midspan. Consistently, PL increases the scraping effect of the blade and it also acts as a divergent for the leakage flow, favouring its recirculation at the tip SS corner and thus reducing the effective passage area available for the core flow (Fig. 13). This, in turn, leads to more radial absolute flow angles at the diffuser inlet, which affect its behaviour (Tab. 4).

\begin{tabular}{ccc}
\hline CASES & $\alpha_{2}\left[{ }^{\circ}\right]$ & Cp [-] \\
\hline PL & 55.74 & 0.514 \\
REF & 58.05 & 0.521 \\
NL & 59.94 & 0.512 \\
\hline
\end{tabular}

\section{Vaneless diffuser flow}

The most striking difference in the vaneless diffuser flow is the radial evolution pattern; as previously discussed, the PL shows a wide region of negative radial velocity at the tip, that is a recirculating flow, detrimental for the diffuser stability (Japikse, 1996).

Fig. 14 shows the evolution for the 3 cases under study, all characterised by the reference flow rate. PL shows higher velocities close to the hub that compensate the wider recirculation; on the contrary, NL shows the more uniform flow conditions, while the reference case is in-between the leaned ones. In tangential direction the non-uniformity is mainly driven by the blade wake mixing.

As the flow evolves in radial direction, the tangential non-uniformity is rapidly recovered, while the one in axial direction is preserved: the tip region for the PL has always the wider backflow. At the hub, the friction on the diffuser wall makes the radial velocity to decrease, leading in the following sections $\left(\mathrm{R}=1.3 \mathrm{R}_{2}\right.$ and $\left.\mathrm{R}=1.59 \mathrm{R}_{2}\right)$ to the onset of a separation bubble. In the last section, the morphology is not that different for the 3 cases: the PL suffers from the initial wider

$\underline{\text { NL case }}$

\section{$R / R_{2}=1.005$}

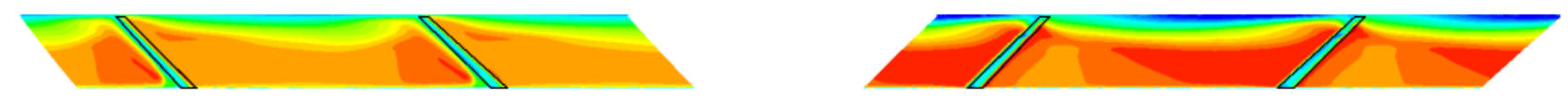

$\mathbf{R} / \mathbf{R}_{\mathbf{2}}=\mathbf{1 . 3 0 0}$

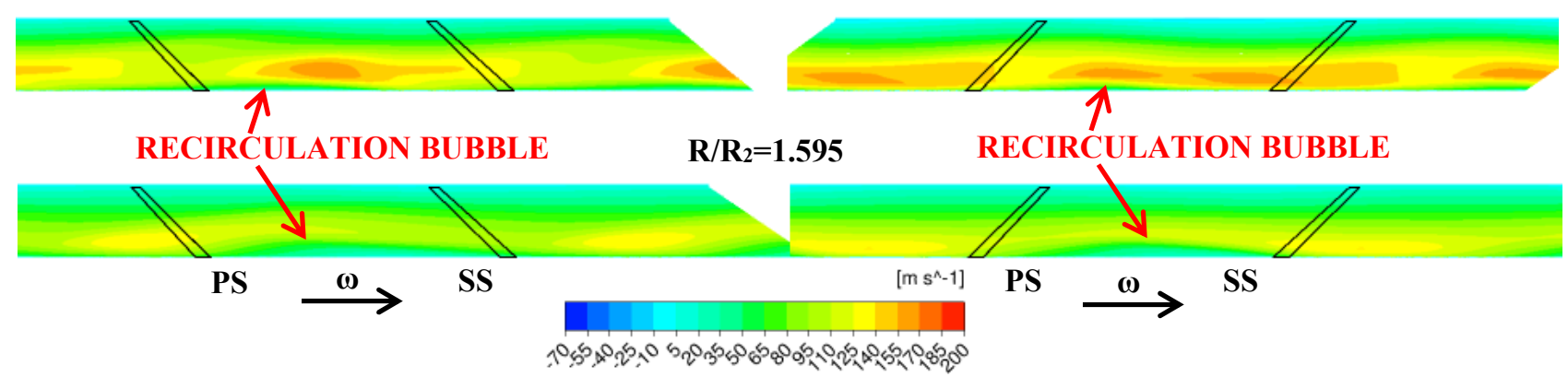

Fig. 14: Radial velocity evolution along the diffuser for the leaned blades 
recirculation region, while the NL from the more tangential flow angle that makes the path longer, and hence the viscous dissipation higher, given the same radial extension.

The discussion on the radial velocity contours matches with the entropy patterns, reported only in two sections for sake of brevity, in Fig. 15. At the diffuser inlet the pattern is dominated by the blade wake and by the dissipations in the tip clearance region. As the flow evolves, a redistribution across the passage is found, and a new region of entropy production is present at the hub, corresponding to the onset of a recirculation bubble for both cases (red arrows).

As for the overall diffuser performances, the averaged flow angle is greater for the NL case than for the PL case. The already-mentioned direct consequence is to have a longer flow path, given the same radial extent, for the NL case that means a slightly lower pressure recovery factor. In the PL case, the shorter path and the larger recirculation allow for a lower pressure recovery, while the REF case is the best trade-off between the two features. Tab. 4 resumes the averaged values for $\alpha_{2}$ and $\mathrm{Cp}$ in the 3 cases under discussion.

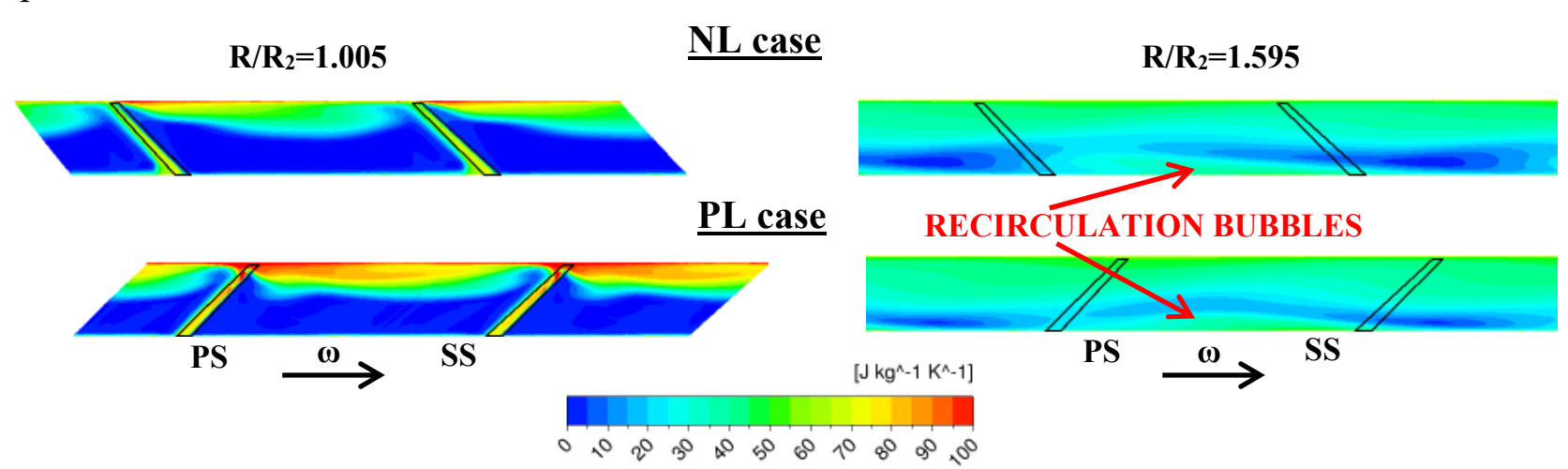

Fig. 15: Entropy variation at the diffuser inlet and outlet for the leaned blades

\section{INFLUENCE OF THE TIP CLEARANCE}

The tip gap reduction effects have been analysed for the reference operating condition, in order to better understand the phenomena occurring in the REF case tip region and their causes. In fact, since the lean geometry affects the blade loading, the tip clearance is expected to couple its effect. The CLO0 case considers a no-clearance faced to a stationary wall in the stationary frame of reference, and for this it is not representative of shrouded impellers but just it consists of a "limit case" for the clearance ones.

The secondary vorticity at the impeller exit for all cases is represented in Fig. 16. As the clearance is reduced, a significant drop in the leakage-related vorticity peak is visible in the upper part of the blade channel. The backflow region close to the tip SS corner is reduced too (Fig. 9 and Fig. 17), which means that its intensity is more affected by the leakage flow decrease rather than by the rotor pressure ratio increase (total-to-total: 2.17 for REF, 2.20 for CL02 and 2.21 for CL00).
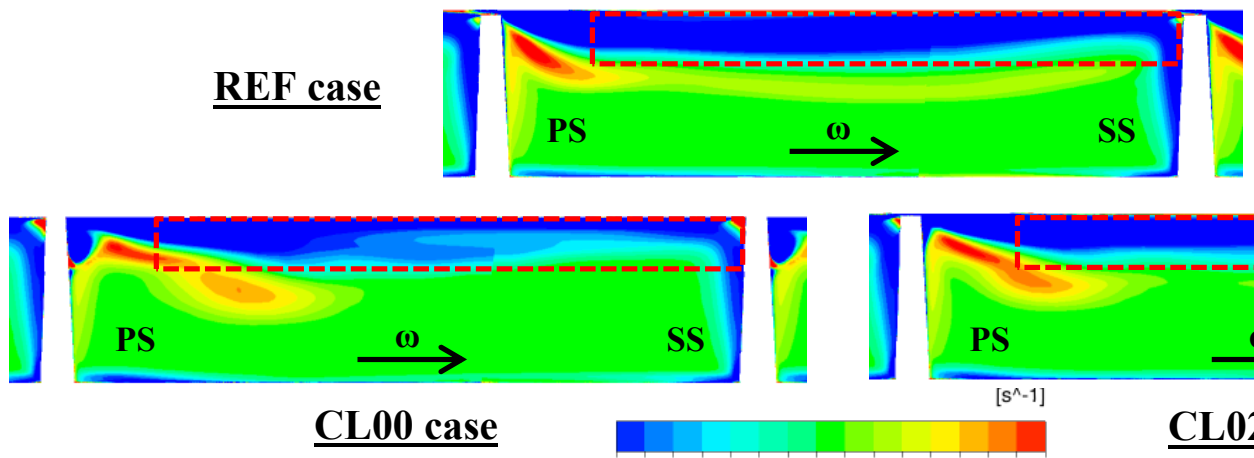

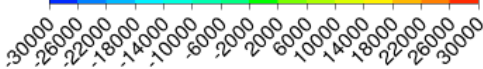

\section{CL02 case}

Fig. 16: Streamwise relative vorticity at $\mathbf{R}_{2}$ for the different clearance cases (red dashed rectangles have the same size) 


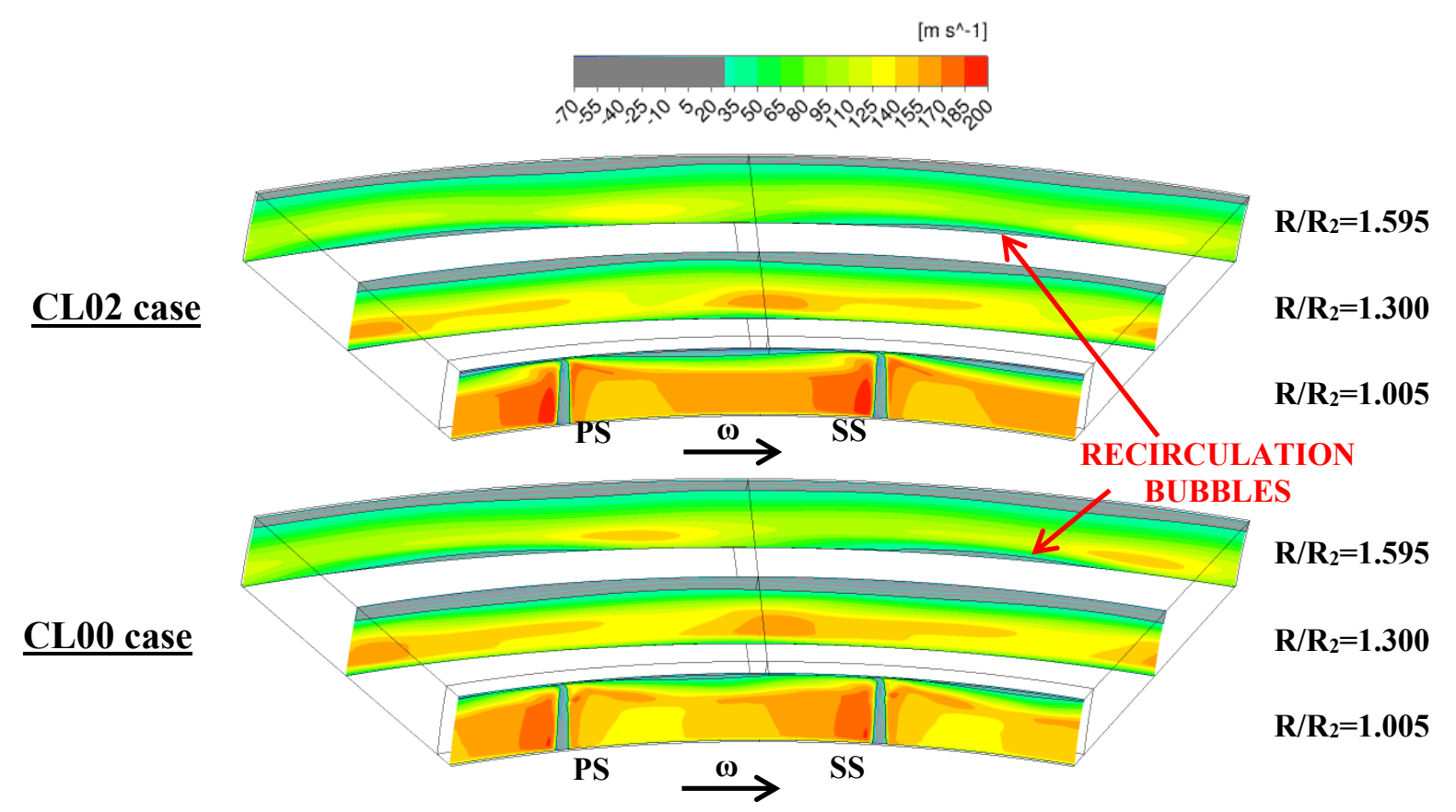

Fig. 17: Radial velocity evolution along the diffuser for the reduced CL cases

As already found by Hong and Abhari (2012), the tip gap reduction has a positive outcome on the impeller efficiency (total-to-total: 95.0\% for REF, 95.8\% for CL02 and 96.5\% for CL00), while its influence seems to be harmful for the vaneless diffuser performance. In fact, the radial velocity contours in Fig. 17 evidence larger recirculation at the hub and casing walls for the CL00 case, leading to a decrease of the overall pressure recovery coefficient, reported in Tab. 5 for all the clearances. For what concerns CL02, it shows an intermediate behaviour between CLO0 and REF cases, both in terms of secondary flow structures and pressure recovery. This result seems somehow surprising: in fact, the clearance allows the recirculation, which promotes turbulent mixing and for this the boundary layer stabilisation. Moreover, the local boundary layer condition in the tip region is to be skewed while passing from the rotating to the stationary frame of reference: this feature contributes to its energisation at the diffuser inlet (the recirculation region and boundary layer have an absolute velocity very close to the peripheral one). Finally, the need of delivering the mass flow makes the average angle to be more

\begin{tabular}{ccc} 
CASES & $\alpha_{2}\left[{ }^{\circ}\right]$ & Cp [-] \\
\hline CLO0 & 61.80 & 0.473 \\
CL02 & 60.07 & 0.502 \\
REF & 58.05 & 0.521 \\
\hline
\end{tabular}

Tab. 5: Overall values for the different clearance cases radial for the REF case; on the contrary, the CL00 case shows a more tangential flow that is more prone to the backflow condition and features a longer path given the same diffuser radial extension (causing higher friction losses and a lower $\mathrm{Cp}$ ).

\section{INFLUENCE OF THE FLOW RATE}

In addition to the previously discussed cases, the two supplementary mass flow rates listed in Tab. 3 have been applied for the reference and leaned geometries.

Fig. 18 and Fig. 19 show that the secondary structures in proximity of the rotor outlet and the radial velocity evolution along the vaneless diffuser present the same main characteristics found for the REF flow rate. However, the MIN flow rate evidences an amplification of the vorticity contours discrepancy among the different geometries close to the casing. This is due to the increase of the tip leakage at lower flow rates, (i.e. at higher incidence angle and loading), which, on one side, makes the PL scraping effect more detrimental and, on the other, makes the NL action of confining the wake fluid at the casing more effective in terms of flow uniformity. In opposition, the MAX flow 


\section{MIN FLOW RATE

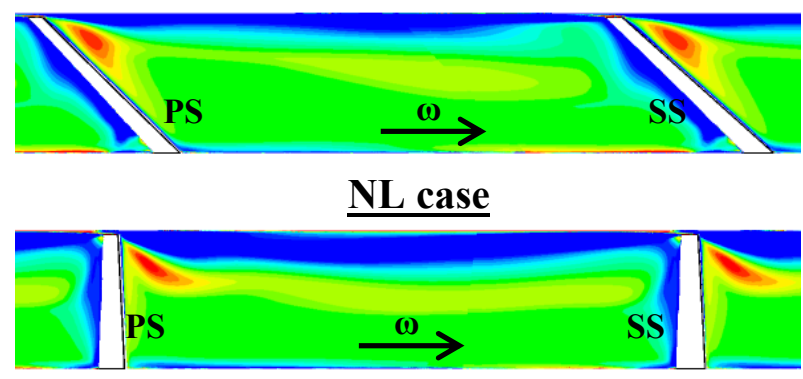

$\underline{\text { REF case }}$

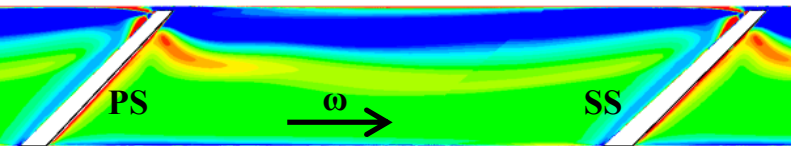

PL case

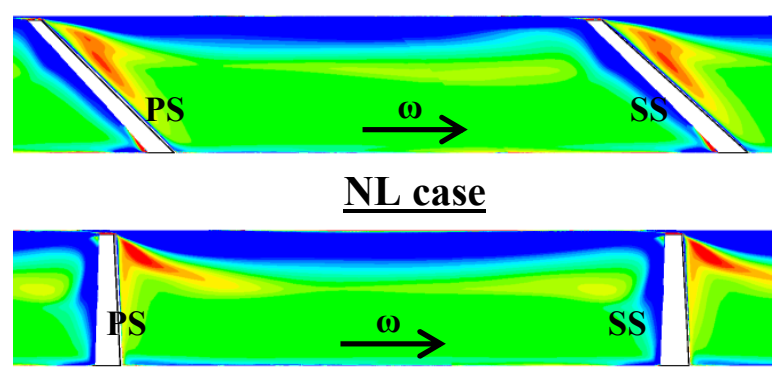

REF case

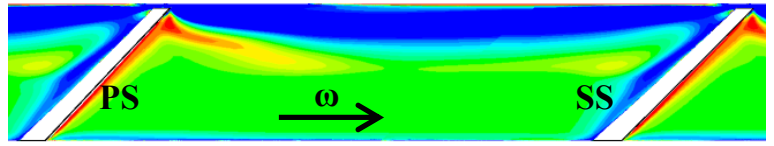

PL case

Fig. 18: Streamwise relative vorticity evolution along the diffuser for the MIN and MAX flow rates
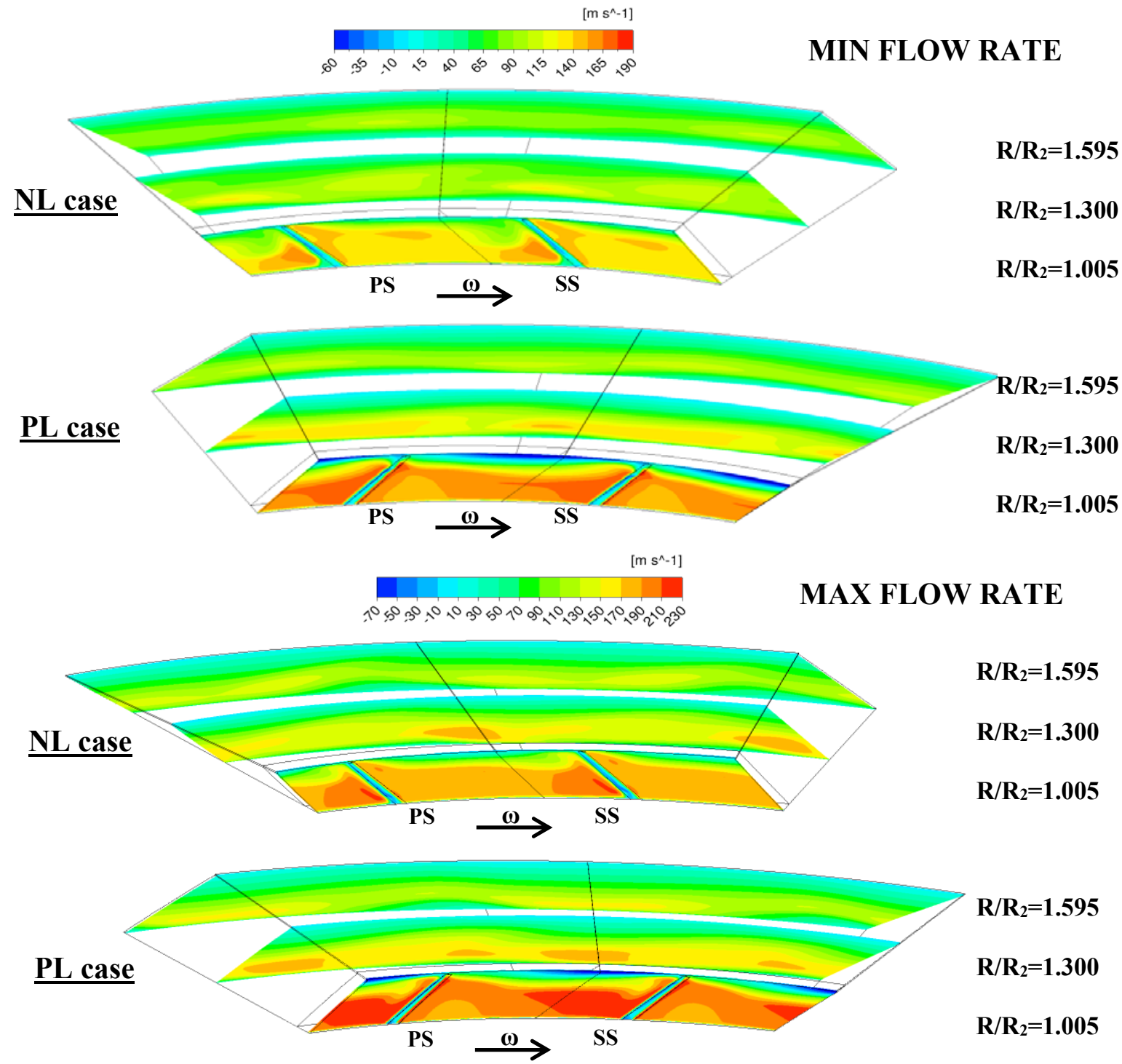

Fig. 19: Radial velocity evolution along the diffuser for the MIN and MAX flow rates 
rate involves a decrease of the tip leakage and consequently a more similar secondary field can be observed for the three blades.

For what concerns the

\begin{tabular}{ccc|ccc}
\hline CASES & $\alpha_{2}\left[^{\circ}\right]$ & Cp [-] & CASES & $\alpha_{2}\left[^{\circ}\right]$ & Cp [-] \\
\hline PL & 59.95 & 0.515 & PL & 51.86 & 0.517 \\
REF & 62.63 & 0.521 & REF & 54.65 & 0.519 \\
NL & 64.45 & 0.527 & NL & 55.94 & 0.515 \\
\hline
\end{tabular}
vaneless diffuser, the overall inlet flow angles and pressure recovery coefficients at MAX and MIN operating conditions

Tab. 6: Overall values for the different cases at MIN (left) and MAX (right) flow rate

are presented in Tab. 6. They show how the average $\alpha_{2}$ is heavily affected by the operating condition, as expected, and the change in the angle is higher when the flow rate reduces $\left(\Delta \alpha_{2} \sim-4.3^{\circ}\right.$ for REF $\rightarrow$ MIN flow rate variation and $\Delta \alpha_{2} \sim 3.7^{\circ}$ for REF $\rightarrow$ MAX). In general, the corresponding change in the $\mathrm{Cp}$ is weak but for the NL case, where for the low flow rate it gains about $4 \%$ as a result of a more uniform inlet flow field.

\section{GLOBAL PARAMETERS}

To gain a global perspective on the effects of lean and flow rate, it seems of interest to discuss the compressor efficiency and pressure ratio: results are shown in Fig. 20.

As the clearance vorticity induced at the impeller outlet by the lean results in a wider and more intense backflow region for the PL geometry, the efficiency shows the lowest values for the PL case in all the flow rate cases, either considering the impeller only or the overall stage. Due to opposite considerations, some beneficial effects of the NL case on the total-to-total impeller efficiency are visible all over the flow rate range. The NL benefits on the impeller are definitively affecting also the overall stage evaluation. In fact, when the total-to-static stage efficiency is of concern, the NL geometry performs well over the whole flow rate range, providing a clear indication of the potential
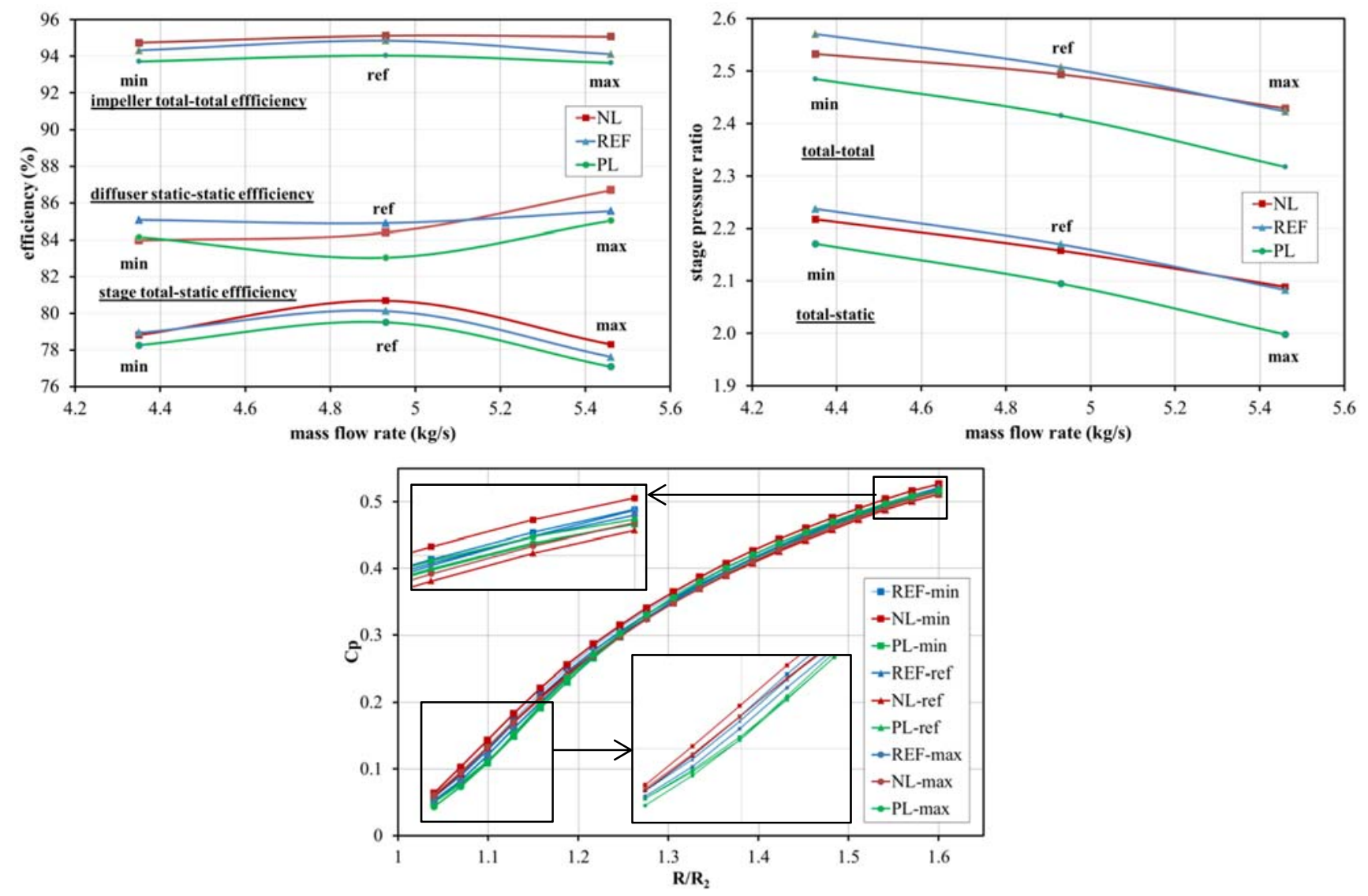

Fig. 20: Efficiency, pressure ratio and $\mathrm{Cp}$ trend for all the cases 
in this design choice. On the side of diffuser static-to-static efficiency, the only conclusion achievable is that the PL never constitutes an interesting option for the analysed machine.

In terms of pressure ratio, the NL and the REF cases provide similar values for all the flow rates, especially at MAX condition, while the PL has always the lowest results. The total pressure curves exhibit good agreement with the ones obtained by He and Zheng (2016). In that study the numerical analysis was performed on a wider range of operating points, suggesting that, on the other hand, PL may push a bit further the surge limit mass flow compared to other cases.

Finally, the PL always provides low $\mathrm{Cp}$ values up to $\mathrm{R}=1.3 \mathrm{R}_{2}$ approximatively. This is the typical radial size of vaneless diffusers in centrifugal compressors, when followed by vaned sections downstream. The opposite can be said for the NL, which shows a better pressure recovery up to $\mathrm{R}=1.3 \mathrm{R}_{2}$ for each of the tested operating conditions. When the diffuser radial extent is $\mathrm{R}>1.3 \mathrm{R}_{2}$, there is no longer a monotonic trend and no general conclusion on the lean effects can be derived, since the action of the viscous stress in the vaneless diffuser seems to smear all the differences and non-uniformities coming from the impeller.

\section{CONCLUSIONS}

The paper discusses the consequences of blade lean and tip clearance on the impeller-diffuser flow and performances for 3 different operating points.

The main effect of the blade lean is related to the clearance vorticity induced at the impeller outlet, that results in a wider, mainly in spanwise direction, and more intense backflow region for the positive lean geometry. This feature results in a higher rotor efficiency for the NL case over the whole flow rate range studied; on the contrary PL is generally the less efficient case.

The influence of lean on the impeller efficiency drives also the total-to-static stage efficiency, which shows the same trend: NL case does better the others, mainly if compared to the PL case, showing always the lowest efficiency.

Furthermore, lean influences the vaneless diffuser pressure recovery up to $R=1.3 \mathrm{R}_{2}$. Such a result appears to be of some importance for designers in the optimisation process and methodology. For a diffuser extension $\mathrm{R}>1.3 \mathrm{R}_{2}$, there is no more a clear trend among the different cases and no general conclusion on the lean effects can be deduced.

Finally, for the considered geometry the rotor tip clearance reduction provides a clear increase in the rotor efficiency, while the effect on the vaneless diffuser is questionable. In fact, as the clearance is reduced the mean flow angle is more tangential and the pressure recovery coefficient decreases, partially counterweighing the efficiency gain accumulated in the impeller.

\section{REFERENCES}

Baines, N.C., Whitfield, A., (1990). Design of radial turbomachines, Longman Scientific \& Technical

Baskharone, E.A., (2006). Principles of turbomachinery in air-breathing engines, Cambridge University press.

Brasz, J.J., (1988). Investigation into the effect of the tip clearance on centrifugal compressors performance, Proceedings of the ASME-IGTI conference, Vol. 1.

Cumpsty, N. A., (1989). Compressor Aerodynamics. Longman Scientific\&Technical, England.

Denton, J.D., Xu, L., (1999). The exploitation of three dimensional flow in turbomachinery design, Proc. of the Institution of Mechanical Engineers, part C: Journal of Mechanical Engineering, Vol. 213.

D'ippolito, G., Dossena, V., Mora, A., (2011). The influence of the blade lean on straight and annular turbine cascade flow field, Journal of Turbomachinery, Vol. 133.

Gaetani, P., Persico, G., Mora, A., Dossena, V., Osnaghi, C., (2011). Impeller - vaned diffuser interaction in a centrifugal compressor at the best efficiency point. ASME GT2011-46223, Proceedings of ASME Turbo Expo 2011: Power for Land, Sea and Air, Vancouver, Canada.

Gaetani, P., Persico, G., Mora, A., Dossena, V., Osnaghi, C., (2012). Impeller-Vaned Diffuser Interaction in a Centrifugal Compressor at Off Design Conditions. J. Turbomach. Vol. 134. 
He, X., Zheng, X., (2016). Mechanism of Lean on the performance of transonic centrifugal compressor impellers, Journal of Propulsion and Power, Vol. 32(5).

Hong, S., Abhari, R.S., (2012). Effect of the tip clearance on impeller discharge flow and vaneless diffuser performance, Proceedings of the institution of Mechanical Engineers, Part A: Journal of Power and Energy, vol. 226(8).

Howard J.H.G., Ashrafizaadeh M., (1994). A Numerical Investigation of Blade Lean Angle Effects on Flow in a Centrifugal Impeller. Proceedings of Turbo Expo: Power for Land, Sea, Air.

Japikse, D., (1996). Centrifugal Compressor Design and Performance. Concepts ETI, Inc.

Krain, H., (1981). A Study on Centrifugal Impeller and Diffuser Flow. Asme J. Eng. Power 103, pp 688-697.

Menter F., Kuntz M., Langtry R.B., (2003). Ten years of industrial experience with the SST turbulence model. Journal of Heat and Mass Transfer, Vol. 4.

Rosic, B., Xu, L., (2012). Blade lean and Shroud leakage flows in low aspect ratio turbines, Journal of Turbomachinery, Vol. 134 (3).

Schleer, M., Hong S.S., Zangene, M., Roduner, C., Ribi, B., Ploger, F., Abhari, R.S., (2004). Investigation of an Inversely Designed Centrifugal Compressor Stage-Part II: Experimental Investigation. Asme Journal of Turbomachinery, Vol. 126, pp 82-90.

Toni, L., Ballarini, V., Cioncolini, S., Gaetani, P., Persico, G., (2010). Unsteady Flow Field Measurements in an Industrial Centrifugal Compressor 39th Turbomachinery Symposium October 4-7, 2010 - Houston, Texas

Van den Braembussche R.A., (2006). Optimization of Radial Impeller Geometry. Design and Analysis of High Speed Pumps, vol.13: pp.1-28.

Ziegler, K.U., Gallus, H., Niehuis, R., (2003) - a. A Study on the Impeller-Diffuser Interaction part I: Influence on the Performance. Journal of Turbomachinery, Vol. 125, Issue 1, pp 173-182

Ziegler, K.U., Gallus, H., Niehuis, R., (2003) - b. A study on the Impeller-Diffuser Interaction part II: Detailed Flow Analysis. Journal of Turbomachinery, Vol. 125, Issue 1, pp 183-192. 